\title{
India introducing routine pneumococcal vaccine
}

\author{
n Cite as: CMAJ 2017 July 17;189:E950. doi: 10.1503/cmaj.1095440
}

n ndia is rolling out the long-awaited pneumococcal conjugate vaccine as part of its government-sponsored immunization program. The vaccine protects against severe forms of pneumococcal diseases, such as pneumonia and meningitis, which kills almost 200000 children in India under the age of five annually, more than any other infectious disease.

"No child should die in the country from vaccine-preventable diseases. We stand committed to reducing child deaths," said Jagat Prakash Nadda, union minister for Health and Family Welfare in announcing the vaccine May 12.

"While these vaccines in the private sector were accessible to only those who could afford them, by making them available under the UIP [Universal Immunisation Programme], the government is ensuring equitable access to those who need them the most, the underprivileged and underserved," Nadda added.

This year, the vaccine will be rolled out to approximately 2.1 million children in Himachal Pradesh and parts of two other states. Eventually the coverage will be expanded across India, but timelines were not provided.

The pneumococcal conjugate vaccine is being introduced with support from Gavi, the Vaccine Alliance, which is providing up to US\$500 million to India's immunization program from 2016 to 2021. Gavi has helped over 50 low-income countries introduce the vaccine into routine immunization programs.

"India is not only the largest Gavi-supported country, but it also has the single
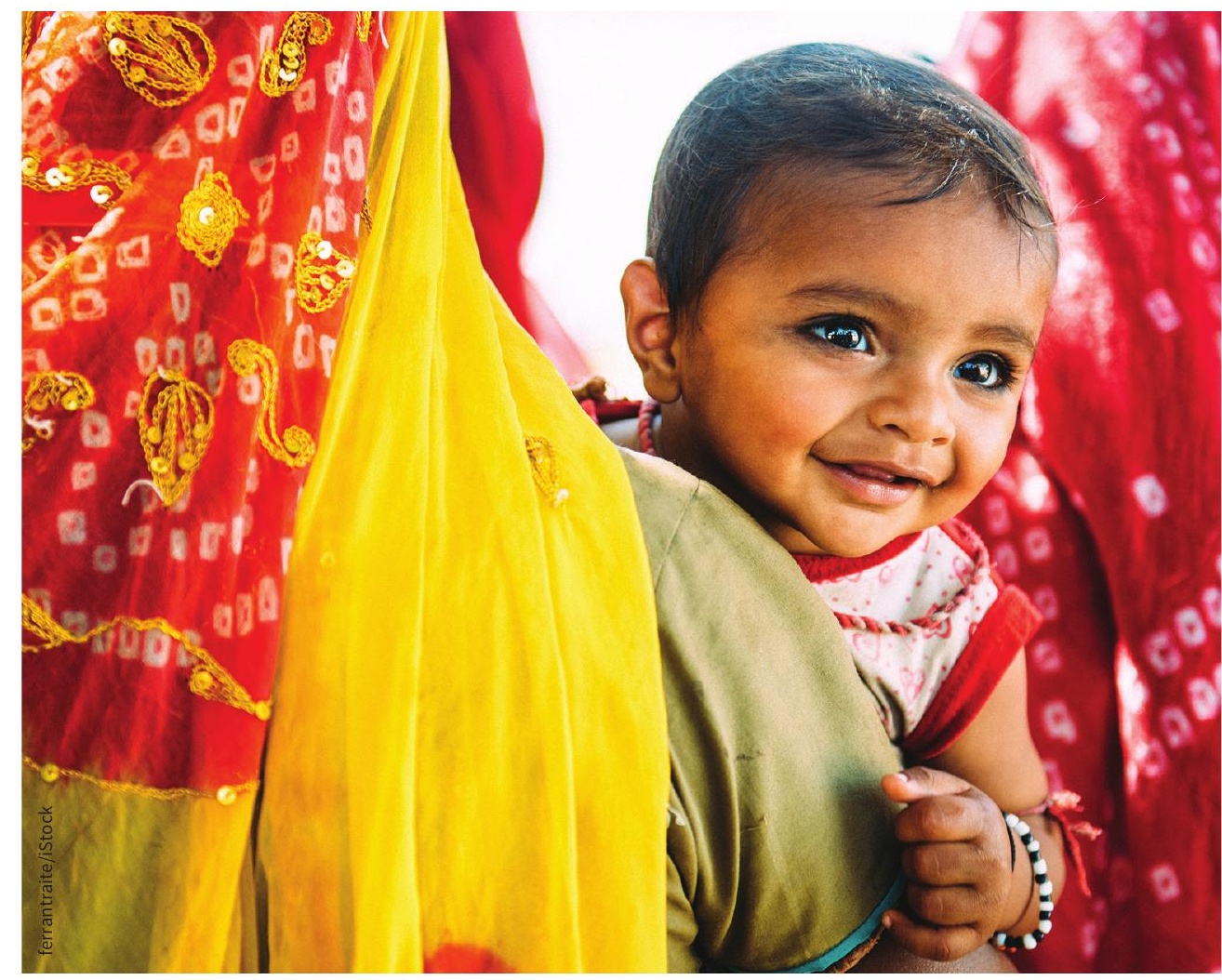

The new routine pneumococcal vaccine in India could eventually save up to 200000 lives annually.

largest number of under-immunized children in the world," said Gavi's CEO Seth Berkley. "This vaccine will save many lives."

Childhood pneumonia accounts for more than $20 \%$ of deaths in childhood, and nearly $20 \%$ of global pneumonia deaths in children under five - the highest number in the world, said Sutapa Agrawal, a Senior Research Scientist and Associate Professor of Epidemiology at the Public Health Foundation of India in New Delhi.
"It affects children in the lower socio-economic sections and in most cases the infection is not detected early," she said.

"We've been trying to introduce the vaccine in the UIP for years to prevent millions of infant deaths in the country," said Dr. Syamal Kumar Bandyopadhyay, a professor of pediatric medicine at Calcutta National Medical College, a public hospital.

Prasun Chaudhuri, Kolkata, India 